\title{
PERCEPCIÓN DE LA VARIABILIDAD PLUVIOMÉTRICA EN LAS POBLACIONES COSTERAS DEL SUR DE LA PROVINCIA DE BUENOS AIRES (ARGENTINA): Una
}

\author{
Propuesta Para el Diseño de Estrategias de Adaptación
}

PERCEPTION OF RAINFALL VARIABILITY IN COASTAL CITIES LOCATED IN THE SOUTH OF

BUENOS AIRES PROVINCE (ARGENTINA): A proposal for the design of adaptation strategies

Dra. M. Luján Bustos

Docente-Investigador. Cátedra: Introducción a la Oceanografía.

Departamento de Geografía y Turismo, Universidad Nacional del Sur.

Dr. Federico Ferrelli

Investigador asistente. Instituto Argentino de Oceanografía (CONICET-UNS)

Ayudante de docencia. Cátedra: Geografía de la Población.

Departamento de Geografía y Turismo, Universidad Nacional del Sur.

\section{RESUMEN}

La región del sudoeste de la provincia de Buenos Aires se caracteriza por presentar una alternancia de eventos secos y húmedos que los actores sociales (AS) perciben en diferentes situaciones cotidianas. El objetivo de este trabajo fue evaluar la percepción de esos AS sobre la variabilidad pluviométrica con el fin de diseñar un plan de manejo sustentable del territorio. Se aplicaron entrevistas y talleres participativos y se analizaron datos diarios, mensuales y anuales de precipitación. Durante un evento seco, los AS observaron, entre otras situaciones, una reducción en el crecimiento de los peces, perjudicando las capturas. Como consecuencia, sugirieron medidas de adaptación tales como vedas más prolongadas. Por otro lado, en un evento lluvioso, identificaron daños en los médanos y playas por las lluvias intensas, perjudicando el desarrollo de actividades turístico-recreativas. Los resultados obtenidos constituyen una fuente de información sólida ya que involucran el conocimiento de la variabilidad pluviométrica junto con la participación activa de AS.

PALABRAS CLAVE: Variabilidad Pluviométrica; Actores Sociales; Percepción; Gestión del Territorio.

\section{ABSTRACT}

The aim of this study was to evaluate the social perception of Stakeholders (SH) about the rainfall variability in cities located in the southwest of Buenos Aires province in order to design a sustainable management plan of the territory. There were applied a series of interviews and participatory workshops with SH and Decision Makers (DM) to understand the social perception, while the rainfall variability was studied with daily, monthly and annual data. The study area was characterized by the alternation of dry and wet events and this situation was perceived by the $\mathrm{SH}$. During a drought, $\mathrm{SH}$ observed a reduction in the fishes growing, harming the catches. As a consequence, they suggested adaptation strategies such as the establishment of long-term closures. On the other hand, in a wet event, the $\mathrm{SH}$ identified damages in dunes and beaches due to the heavy rains, damaging the development of tourist-recreational activities. The results allowed to contribute a solid data source since they involved the study of rainfall variability and the $\mathrm{SH}$ and DM perception.

Keywords: Rainfall variability; Stakeholders; Perception; Sustainable land management plan.

Publicado en formato digital: Dra. M. Luján Bustos y Dr. Federico Ferrelli. PERCEPCIÓN DE LA VARIABILIDAD PLUVIOMÉTRICA EN LAS POBLACIONES COSTERAS DEL SUR DE LA PROVINCIA DE BUENOS AIRES (ARGENTINA): Una Propuesta Para el Diseño de Estrategias de Adaptación. Revista Geográfica Digital. IGUNNE. Facultad de Humanidades. UNNE. Año 15. № 30. Julio - Diciembre 2018. ISSN 1668-5180 Resistencia, Chaco.

En: http://hum.unne.edu.ar/revistas/geoweb/default.htm 
Revista Geográfica Digital. IGUNNE. Facultad de Humanidades. UNNE. Año 15. № 30.

Julio - Diciembre 2018. ISSN 1668-5180 Resistencia, Chaco

\section{INTRODUCCIÓN}

Debido a los efectos de la variabilidad pluviométrica sobre la población de distintas aglomeraciones urbanas y su relación con El Niño Oscilación Sur (ENOS), que se ha intensificado en las últimas décadas (IPCC, 2013, UNISDR, 2011), los informes del Panel Internacional sobre Cambio Climático (IPCC) han remarcado la importancia de estudiar los impactos del ENOS considerando la percepción de los habitantes de un área (Magrin et al., 2007). El estudio de la variabilidad climática en las zonas costeras, con el objetivo de generar planes de mitigación y adaptación, adquirió relevancia, particularmente en Argentina. Este país tiene una de las costas más extensas de América Latina. Estas zonas están sujetas a la erosión, con un retroceso de la línea de costa del orden de 3-5 m por año debido a los procesos de deriva litoral (Pratolongo et al., 2006) y fuertes tormentas (Merlotto y Bértola, 2006; Bustos et al., 2009; Huamantinco Cisneros et al., 2011).

La costa sudoeste de la provincia de Buenos Aires (Fig. 1) se caracteriza por presentar una amplia gama de condiciones geomorfológicas, físicas y socio-económicas que la hacen única en la costa argentina. En esta área se emplaza un polo industrial petroquímico y petrolero. Además, la pesca artesanal y el turismo, directamente relacionados con la costa y las playas, son las actividades económicas más importantes, luego de la actividad industrial.

En clima de la región expone a las localidades ubicadas en estas costas a los efectos de la variabilidad climática ya que la alternancia de periodos secos y húmedos generan procesos de largas sequias e inundaciones que afectan significativamente su desarrollo socio-económico y las condiciones ambientales. En este contexto es que la percepción de la población y, particularmente, el concepto de desarrollo local han surgido como un modelo de acción estratégico para mitigar los problemas que enfrenta la comunidad teniendo en cuenta los recursos propios del territorio. Este proceso incluye las acciones destinadas a resolver problemas concretos que mejoren la calidad de vida de los habitantes. La participación activa de los Actores Sociales (AS) aporta la información necesaria para la re-planificación del espacio con el fin de mejorar las condiciones ambientales para su bienestar presente y futuro (Barton et al., 2009). Por ello, el objetivo de este trabajo fue evaluar la percepción de los AS sobre la variabilidad pluviométrica durante dos eventos diferentes (seco y húmedo) en las localidades costeras del sudoeste de la provincia de Buenos Aires con el fin de adquirir información necesaria para la generación de un plan de manejo sustentable del territorio que permita generar estrategias de adaptación para reducir los impactos negativos que estos eventos tienen en el área.

Figura 1. Área de estudio en la costa sudoeste de la provincia de Buenos Aires.

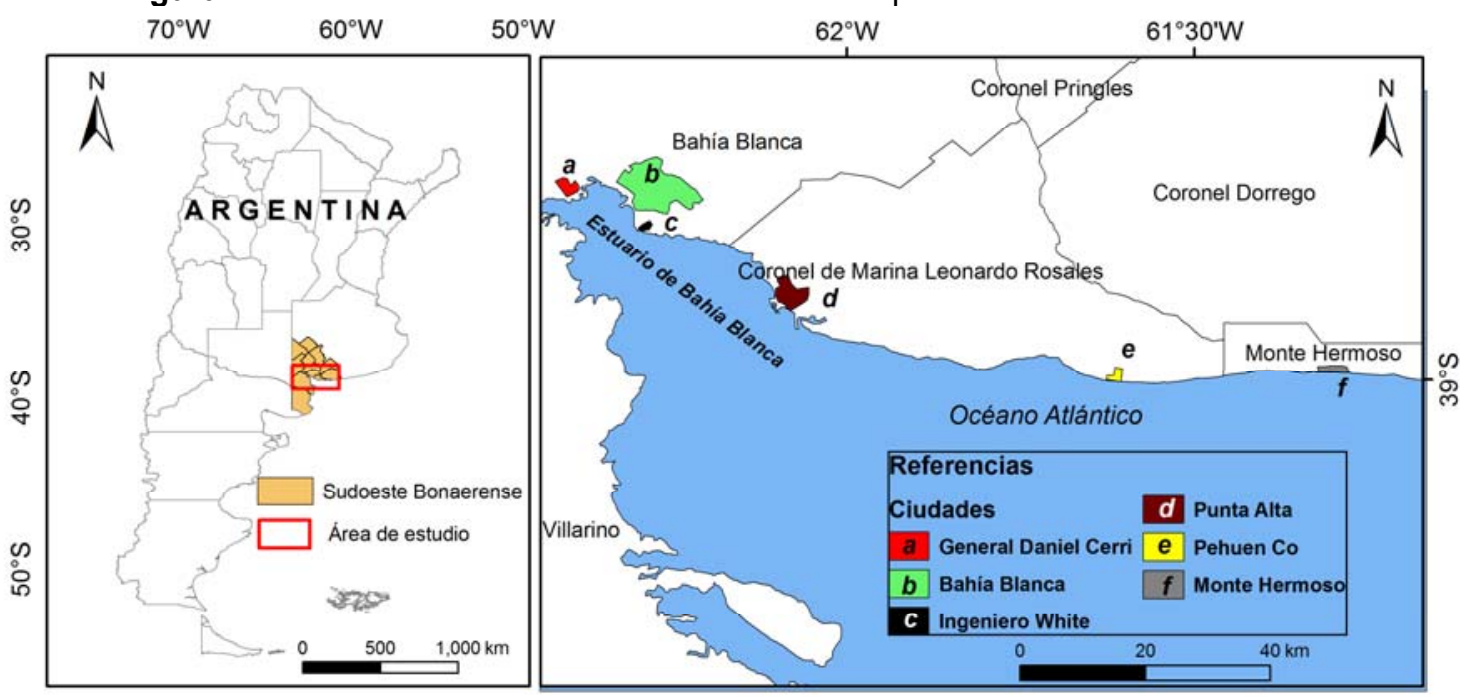

Publicado en formato digital: Dra. M. Luján Bustos y Dr. Federico Ferrelli. PERCEPCIÓN DE LA VARIABILIDAD PLUVIOMÉTRICA EN LAS POBLACIONES COSTERAS DEL SUR DE LA PROVINCIA DE BUENOS AIRES (ARGENTINA): Una Propuesta Para el Diseño de Estrategias de Adaptación. Revista Geográfica Digital. IGUNNE. Facultad de Humanidades. UNNE. Año 15. No 30. Julio - Diciembre 2018. ISSN 1668-5180 Resistencia, Chaco.

En: http://hum.unne.edu.ar/revistas/geoweb/default.htm 
Revista Geográfica Digital. IGUNNE. Facultad de Humanidades. UNNE. Año 15. № 30.

Julio - Diciembre 2018. ISSN 1668-5180 Resistencia, Chaco

\section{Área de estudio}

El área de estudio se centra en la costa sudoeste de la provincia de Buenos Aires (Argentina) a lo largo de aproximadamente $100 \mathrm{~km}$ de longitud de este-oeste. La división administrativa define tres partidos diferentes (Fig. 1). De oeste a este se agrupan: en el partido de Bahía Blanca las localidades de General Daniel Cerri, Bahía Blanca (la localidad de mayor tamaño y cabecera del partido) e Ingeniero White; el partido de Coronel de Marina Leonardo Rosales, en donde se emplazan las localidades de Villa del Mar y Pehuen Co y en el partido de Monte Hermoso se consideró la ciudad homónima (Fig. 1).

El clima de la región es templado, con vientos que prevalecen del sector noroeste. Las heladas son comunes durante los inviernos. La variabilidad de la lluvia es fuerte y se ve afectada por la alternancia de eventos secos y húmedos, siendo estos los más intensos de toda la Región Pampeana. Las precipitaciones son frecuentes, de larga duración y baja intensidad. La estacionalidad térmica permite la diferenciación de veranos a inviernos y la pluviométrica, estaciones secas y húmedas (Aliaga et al., 2017). En general, las precipitaciones ocurren durante la primavera y el verano, son menos intensas en el otoño y se hacen más escasas hacia el invierno (Ferrelli et al., 2017). En el área de estudio el gradiente pluviométrico decrece en sentido noroeste-sudoeste. Las precipitaciones son altamente dependientes de las condiciones sinópticas y tienen una gran variabilidad espacio-temporal. La misma es consecuencia de fenómenos de escala global como El Niño Oscilación Sur (Bohn et al., 2016; Ferrelli et al., 2017) y otros de escala local como la influencia orográfica (Aliaga et al., 2017).

El área se puede subdividir en dos tipos si se consideran sus características de sitio y localización. La primera es la costa de la zona del estuario de Bahía Blanca y la segunda son las costas de la zona externa del estuario, con menos influencia del mismo. El estuario de Bahía Blanca presenta una serie de grandes canales de marea con dirección noroeste-sureste separados por extensas planicies de marea, marismas e islas. Una gran porción del estuario corresponde a una reserva natural y está habitada. Sobre la costa norte se encuentra el canal de mayor tamaño denominado "Canal Principal". Este contiene el sistema portuario de aguas profundas más grande de Argentina. Debido a la falta de entrada de sedimentos desde el continente y desde la plataforma interna adyacente, el estuario se encuentra en una fuerte condición de erosión. Perillo (1997) pronosticó que, con un aumento de 1,5 m en el nivel medio del mar, todo el estuario podría estar permanentemente cubierto por el mar con la pérdida del mayor humedal costero del país.

El Segundo tipo de costa comprende las localidades de Monte Hermoso y Pehuen Co (Fig. 1). Estas centran su actividad económica en el turismo de sol y playa. Ambas tienen playas de arena de similares características, pero su desarrollo ha sido diferente. Monte Hermoso se convirtió en una ciudad que edificó sobre el sistema de médanos frontales. En cambio, Pehuen Co es una localidad pequeña que ha intentado preservar el entorno natural. Sus calles están sin pavimentar y con abundante arbolado introducido (Bustos et al., 2016). Asimismo, la erosión costera es un problema importante en ambas playas. En Monte Hermoso, este proceso surge debido a la presencia de elementos antrópicos. Los edificios reemplazaron la primera línea de médanos y facilitaron los procesos de erosión. Después de la ocurrencia de tormentas severas, la contribución natural del sedimento sobre este sector no es suficiente para recuperar todo el sedimento que se está perdiendo (Huamantinco, 2012). Pehuen Co tiene una erosión natural al estar en una saliente de la costa. Esta erosión se suma a la presión antrópica propia de la urbanización (Bustos et al., 2017).

\section{MATERIALES Y MÉTODOS}

Se realizó un estudio continuo de la percepción de la población en los años 2012 y 2017 . El primero de ellos reflejó condiciones de sequía y el segundo de precipitaciones abundantes. En este estudio, se analizaron por un lado la percepción social de la variabilidad pluviométrica y, por el otro, se evaluaron las condiciones pluviométricas mediante el análisis estadístico de datos climáticos. Para realizar las actividades de recolección de información social, se organizaron los talleres en distintos grupos. Esto se realizó debido a la existencia de diferentes características socio-ecológicas en cada

Publicado en formato digital: Dra. M. Luján Bustos y Dr. Federico Ferrelli. PERCEPCIÓN DE LA VARIABILIDAD PLUVIOMÉTRICA EN LAS POBLACIONES COSTERAS DEL SUR DE LA PROVINCIA DE BUENOS AIRES (ARGENTINA): Una Propuesta Para el Diseño de Estrategias de Adaptación. Revista Geográfica Digital. IGUNNE. Facultad de Humanidades. UNNE. Año 15. № 30. Julio - Diciembre 2018. ISSN 1668-5180 Resistencia, Chaco.

En: http://hum.unne.edu.ar/revistas/geoweb/default.htm 
pueblo, por ello, se formó un grupo con los AS provenientes de las localidades del estuario (General Daniel Cerri, Ingeniero White y Villa del Mar) y otro con las costeras (Pehuen Co y Monte Hermoso). Esta división permitió generar una mejor comprensión de la percepción de los AS. Ello, junto con la ocurrencia de períodos pluviométricamente diferentes facilitó la obtención de información sobre la percepción de los AS en cada uno de estos eventos y sus propuestas de acción para el futuro.

Los datos se trabajaron de forma cuali-cuantitativa. Para obtener y analizar las precipitaciones se utilizaron datos del Servicio Meteorológico Nacional (SMN, Argentina) y para la percepción se realizaron talleres, y entrevistas siguiendo la metodología representada en la Figura 2.

Figura 2. Pasos metodológicos considerados para el análisis de la percepción social sobre la variabilidad pluviométrica.

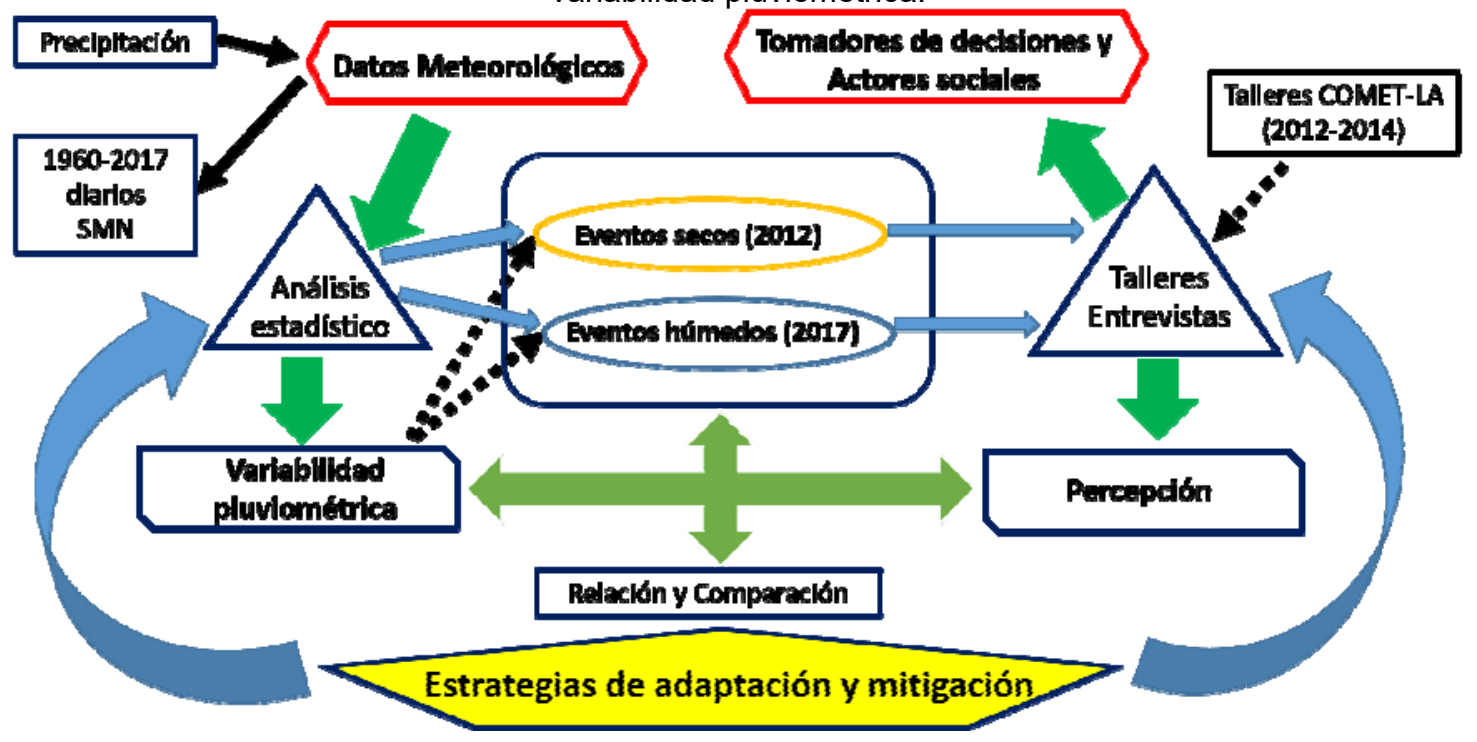

\section{Análisis de la percepción}

En el marco del Proyecto COMET-LA se realizaron entre 2012 y 2015 diferentes actividades que permitieron detectar las características socio-ecológicas en el área de estudio y los impactos que la variabilidad climática produjo en ellas. Durante el primer semestre de 2012, se convocó a los AS y Tomadores de Decisiones (TD) más representativos de las comunidades para participar en varios talleres. Los mismos, junto con las entrevistas, estuvieron basados en metodologías participativas de trabajo. El objetivo de estas actividades fue recopilar información sobre la percepción social a cerca de los problemas ambientales y sus consecuencias socioeconómicas en el área de estudio.

Los criterios utilizados para seleccionar a los informantes clave para participar en los talleres fueron la pertinencia y la representatividad local (London et al., 2012). El primero fue evaluado por las relaciones entre los usuarios y los recursos y su capacidad para afectar el sistema socio-ecológico, así como su conocimiento sobre el mismo. El segundo se definió de acuerdo con el nivel de qué tan bien o con qué precisión alguien reflexiona sobre el grupo (Rojas et al., 2014). De esta forma, los participantes de estos talleres fueron pescadores, bomberos, guardavidas, dueños de locales comerciales (bares, restaurantes, etc.), delegados municipales, entre otros. La concurrencia en cada taller varió entre 6 y 15 participantes.

Una debilidad relevante fue la falta de experiencias participativas de la comunidad. Por esta razón, los talleres comenzaron con una explicación sobre la variabilidad climática seguida de una técnica de lluvia de ideas que permitía obtener datos sin juicio de valor previo o información filtrada. El resultado

Publicado en formato digital: Dra. M. Luján Bustos y Dr. Federico Ferrelli. PERCEPCIÓN DE LA VARIABILIDAD PLUVIOMÉTRICA EN LAS POBLACIONES COSTERAS DEL SUR DE LA PROVINCIA DE BUENOS AIRES (ARGENTINA): Una Propuesta Para el Diseño de Estrategias de Adaptación. Revista Geográfica Digital. IGUNNE. Facultad de Humanidades. UNNE. Año 15. No 30. Julio - Diciembre 2018. ISSN 1668-5180 Resistencia, Chaco.

En: http://hum.unne.edu.ar/revistas/geoweb/default.htm 
fue la aparición de ideas inesperadas que se integraron en una lista para ser empleadas en las siguientes etapas. Geilfus (2002) indicó que una de las ventajas de la lluvia de ideas es recopilar opiniones y percepciones de un amplio y variado grupo de personas. La aplicación de esta técnica fue apropiada para preguntas sobre la vida de la comunidad y las percepciones y reacciones del grupo individual o social. En general, el intercambio de ideas tuvo cuatro pasos: 1) introducción de la pregunta, problema o tema (oral y escrito); 2) invitación a los participantes a responderlas; 3) escritura de las respuestas en una hoja de papel y 4) análisis y promoción de la discusión o soluciones a los problemas mencionados empleando la lista confeccionada (London et al., 2012; Rojas et al., 2014). En 2016 y 2017 se entrevistó de forma personal a cada uno de los AS y TD que participaron de los talleres en los años previos. A cada uno se le consultó sobre la percepción de ellos sobre la posible variación en las precipitaciones y sus propuestas para solucionar los problemas que ello ocasionaba.

\section{Variabilidad pluviométrica}

Los datos de precipitación se obtuvieron de una estación meteorológica ubicada en la ciudad de Bahía Blanca (Fig. 1) perteneciente al Servicio Meteorológico Nacional para el período 1960-2017. En una primera instancia, se realizó un testeo de la calidad de los datos con el software RClimDex (Zang y Yang, 2013) y se evaluó la homogeneidad con el RHTest V4 (Wang et al., 2010). El porcentaje de datos faltantes y de valores atípicos fue de $1,2 \%$ y $0,1 \%$, respectivamente.

Los análisis meteorológicos se realizaron en diferentes escalas de tiempo. Por un lado, con datos diarios, se calcularon 10 índices pluviométricos extremos definidos por el Equipo de Expertos en Detección e Índices de Cambio Climático (ETCCDI) con el objetivo de caracterizar las tormentas, los días lluviosos, etc. en 2012 y 2017. Estos índices proporcionaron una visión detallada del comportamiento de la precipitación y han sido aplicados en distintas partes del mundo con éxito (Peterson et al., 2001; Zhang et al., 2011). Del listado original, en este trabajo se aplicaron los siguientes: i) Rx1day. Indica la máxima precipitación ocurrida en un día, ii) Rx5days. Precipitación máxima ocurrida durante cinco días consecutivos, iii) R10. Tormentas fuertes, iv) R20. Tormentas muy fuertes, v) Rnn. Tormentas extremadamente fuertes, vi) R95p. Días muy húmedos, vii) R99p. Días extremadamente húmedos, viii) PRCPTOT. precipitación total ocurrida en los días húmedos, es decir, en aquellos en donde se registró más de $1 \mathrm{~mm}$ por día, ix) CDD. Días secos consecutivos y $\mathrm{x}$ ) CWD. Días húmedos consecutivos.

Por otro lado, los datos se analizaron en escalas de tiempo mensual, estacional y anual. Se aplicó el Índice Estandarizado de Precipitación (IEP) (Edwards et al., 1997). Las series de tiempo mensuales para un período específico son necesarias para calcularlo. Este índice ha sido recomendado por la Lincoln Declaration on Drought Indices (Hayes et al., 2011) y es el más adecuado para estudiar los períodos secos en América del Sur (Penalba y Ribera, 2016). Se ha aplicado en todo el mundo con el objetivo de analizar las condiciones futuras de sequía (Orlowsky y Sereviratne, 2013; Aliaga et al., 2017).

EI IEP se aplicó considerando escalas temporales de 1, 3 y 12 meses, ya que se buscó representar los períodos de sequía y humedad a corto y largo plazo (mensuales, estacionales y anuales), respectivamente (Aliaga et al., 2017). Al aplicar el IEP en estas escalas temporales fue posible identificar la intensidad del evento (anual y estacional) y su duración en meses. Los períodos secos se identificaron cuando los valores fueron menores a -1,0, lo que significa que las precipitaciones de ese evento fueron 1 desvío estándar menor. Por otro lado, los húmedos fueron aquellos cuyos valores fueron superiores a 1 (Aliaga et al., 2017).

Finalmente, se realizó un análisis de las precipitaciones a escala anual. Se aplicaron los métodos de Quintiles (Bolognesi 1971) y Normal para determinar los años secos, normales y húmedos (Ferrelli et al., 2012). Los quintiles se identificaron como Q1, Q2, Q3 y Q4 (Tabla 2), siguiendo la siguiente fórmula:

$$
Q_{k}=\frac{k_{n}}{5}
$$

donde $\mathrm{k}$ es el orden del quintil $(\mathrm{k}=1,2,3,4)$ y $\mathrm{n}$ es el número de observaciones. La clasificación de los resultados se presenta en la Tabla 1.

Publicado en formato digital: Dra. M. Luján Bustos y Dr. Federico Ferrelli. PERCEPCIÓN DE LA VARIABILIDAD PLUVIOMÉTRICA EN LAS POBLACIONES COSTERAS DEL SUR DE LA PROVINCIA DE BUENOS AIRES (ARGENTINA): Una Propuesta Para el Diseño de Estrategias de Adaptación. Revista Geográfica Digital. IGUNNE. Facultad de Humanidades. UNNE. Año 15. № 30. Julio - Diciembre 2018. ISSN 1668-5180 Resistencia, Chaco.

En: http://hum.unne.edu.ar/revistas/geoweb/default.htm 
Revista Geográfica Digital. IGUNNE. Facultad de Humanidades. UNNE. Año 15. № 30.

Julio - Diciembre 2018. ISSN 1668-5180 Resistencia, Chaco

Tabla 1. Clasificación de periodos anuales de precipitaciones según el Método de Quintiles (Bolognesi, 1971). $\mathrm{P}$ = precipitación anual.

\begin{tabular}{|l|l|}
\hline Año muy seco & $P<1 \mathrm{Q}$ \\
\hline año seco & $1 \mathrm{Q} \leq \mathrm{P}<2 \mathrm{Q}$ \\
\hline Año normal & $2 \mathrm{Q} \leq \mathrm{P}<3 \mathrm{Q}$ \\
\hline Año húmedo & $3 \mathrm{Q} \leq \mathrm{P}<4 \mathrm{Q}$ \\
\hline año muy húmedo & $\mathrm{P} \geq 4 \mathrm{Q}$ \\
\hline
\end{tabular}

\section{RESULTADOS}

\section{Percepción de los actores sociales sobre la variabilidad pluviométrica}

Los talleres y las entrevistas mostraron diferencias entre las opiniones de AS de las diferentes ciudades para ambos periodos analizados (2012 y 2017). En las localidades ubicadas en las costas del estuario (General Daniel Cerri, Ingeniero White y Villa del Mar), el principal problema se centró en la disminución de las pesquerías debido a la contaminación del agua y al dragado del Canal Principal del estuario y no a la variabilidad climática. Por otro lado, en las ciudades costeras (Monte Hermoso y Pehuen Co), los problemas se centraron en la pesca y el turismo, principalmente en relación con las variaciones de temperatura, las rotaciones del viento y la alternancia de eventos secos y húmedos. En Pehuen Co, los mayores problemas fueron en torno a la erosión del médano frontal. La mayoría de los cambios percibidos se atribuyeron a la acción antropogénica.

En el primer período de estudio, los AS observaron un aumento en la frecuencia e intensidad de los períodos de sequía, especialmente los bomberos. Las condiciones de sequía y la tala de bosques, según su apreciación, generaron la ocurrencia de fuertes tormentas de arena y aumentos de incendios. Al igual que los bomberos, los guardavidas notaron los cambios de las tormentas durante 2012. Agregaron que "en el pasado cerrábamos la playa por las fuertes tormentas de verano y ahora no lo hicimos". Además, los guardavidas detectaron un número significativo de canales y barras en la costa que hicieron de la playa un lugar más peligroso para los nadadores. Un guardavida dijo: "Se necesitan sudestadas en verano para alisar la playa", esto indico claramente la falta de tormentas fuertes que erosionen la playa.

En el 2017, durante el periodo húmedo la mayoría de los AS, percibieron una mayor cantidad de "Sudestadas" (fuertes tormentas del sudeste) y tormentas de verano de menor intensidad pero frecuentes. En el caso de Pehuen Co y Monte Hermoso, la erosión y el problema relacionado a ello (avance del agua hacia las construcciones de la costa) se hizo más intenso. Los AS en Monte Hermoso se mostraron preocupados pero no manifestaron que fuese un problema del cual hubiera que ocuparse al corto plazo. En cambio, en Pehuen Co, debido a la erosión a causa de las sudestadas durante 2017, se derrumbaron construcciones sobre el médano frontal lo que generó que los AS pudiesen ver el problema como algo urgente e importante. Un comerciante expreso su preocupación diciendo: "si no hacen algo ya el agua se llevará todas las casas bonitas del frente costero y eso nos perjudicará a todos porque nadie quiere vacacionar en un lugar donde hay casas derrumbadas".

En tanto, en las localidades del estuario, los AS percibieron el aumento de precipitaciones acompañadas de tormentas eléctricas fuertes. Lo percibieron por la cantidad de veces que se anegaron las calles y los accidentes a causas de rayos. Algunos pescadores expresaron la variación explicando: "tenemos ejemplares de peces que no son de esta época y aparecen en las redes ahora, algo está cambiando".

Publicado en formato digital: Dra. M. Luján Bustos y Dr. Federico Ferrelli. PERCEPCIÓN DE LA VARIABILIDAD PLUVIOMÉTRICA EN LAS POBLACIONES COSTERAS DEL SUR DE LA PROVINCIA DE BUENOS AIRES (ARGENTINA): Una Propuesta Para el Diseño de Estrategias de Adaptación. Revista Geográfica Digital. IGUNNE. Facultad de Humanidades. UNNE. Año 15. № 30. Julio - Diciembre 2018. ISSN 1668-5180 Resistencia, Chaco.

En: http://hum.unne.edu.ar/revistas/geoweb/default.htm 


\section{Variabilidad pluviométrica}

El área de estudio tiene una precipitación media anual de $660 \mathrm{~mm}$ anuales $\pm 144,6 \mathrm{~mm}$. Los eventos secos y húmedos son recurrentes (Figura $3 \mathrm{~A}$ ). Las precipitaciones tienen una clara estacionalidad, siendo mayores en el verano y menores en el otoño e invierno. Específicamente el período 20122017, correspondió a un evento seco en 2012 (también identificado con el método de Quintiles). Su duración fue de 13 meses, seguido por uno húmedo que ocurrió desde septiembre de 2014 y se extendió durante todo el año 2015 (16 meses). Finalmente, desde febrero de 2016 y hasta diciembre de 2017, se presentó un evento normal (Según el IEP y el método de Quintiles) (Figura 3A).

El año 2012 se caracterizó por ser un año seco (IEP $=-1,4)$, en donde el verano y el invierno fueron las estaciones más secas. Durante ese año se registraron 490,6 mm anuales, es decir 169,4 mm menos que la precipitación media anual esperada para la región. Por otro lado, en 2017 se presentó un año normal, sin embargo, se observó un verano muy lluvioso (IEP $=1,5$ ) y una primavera seca $($ IEP $=-1,1)$. Las precipitaciones estivales acumularon $290,4 \mathrm{~mm}$, es decir, $90 \mathrm{~mm}$ por encima de lo esperado. En la primavera, precipitaron $85 \mathrm{~mm}$ menos. En este año se registraron $671,2 \mathrm{~mm}$ de precipitación anual, es decir, 11,2 por encima del valor medio anual (Figura 3B).

En 2012, el número de días secos consecutivos fue el mayor de los últimos 10 años, contabilizando 46. Este hecho generó que las condiciones ambientales sean propensas para que se intensifiquen los procesos de erosión eólica. Por otro lado, en 2017, se observó un evento normal, con un verano muy lluvioso, en donde las precipitaciones fueron hasta $100 \mathrm{~mm}$ mayores que las registradas en el verano $(200,1 \mathrm{~mm})$ en el área de estudio. Durante esta estación, se registraron 12 tormentas torrenciales, lo que favoreció las condiciones para que se generen anegamientos y erosión hídrica en las costas dado que en estos eventos las precipitaciones superaron los $25 \mathrm{~mm}$ diarios (Tabla 2).

Figura 3. Análisis de los períodos secos y húmedos para el área de estudio. A. Período 1960-2017, B. Período 2012-2017.

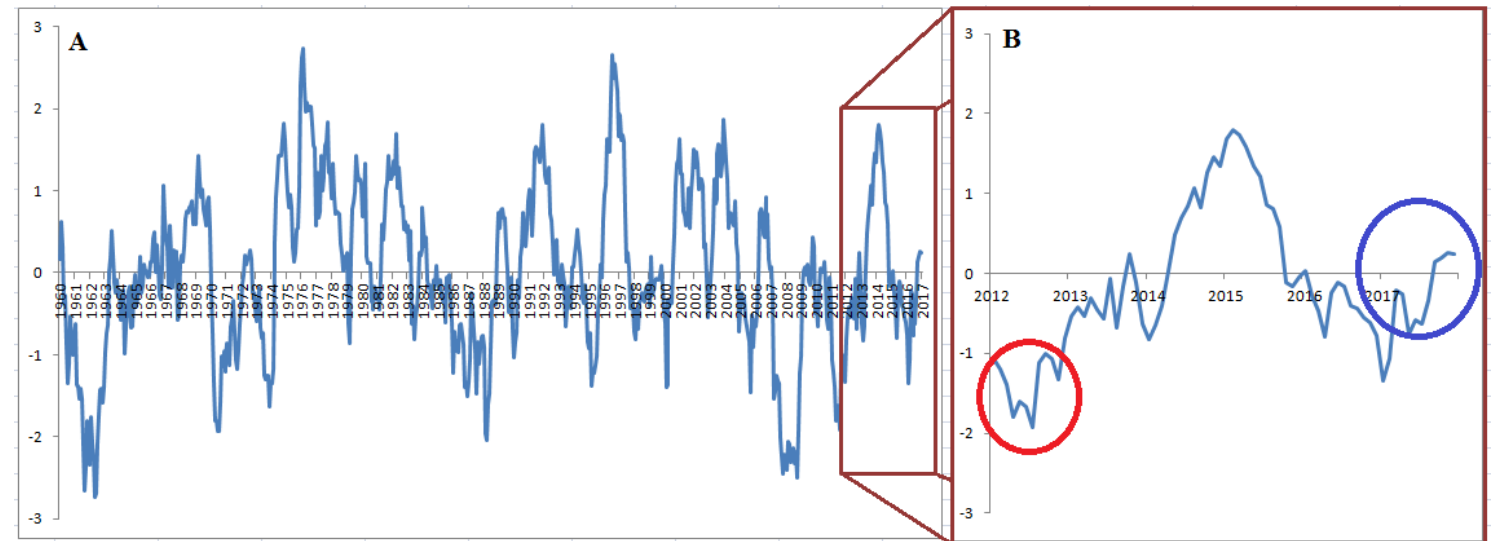

Publicado en formato digital: Dra. M. Luján Bustos y Dr. Federico Ferrelli. PERCEPCIÓN DE LA VARIABILIDAD PLUVIOMÉTRICA EN LAS POBLACIONES COSTERAS DEL SUR DE LA PROVINCIA DE BUENOS AIRES (ARGENTINA): Una Propuesta Para el Diseño de Estrategias de Adaptación. Revista Geográfica Digital. IGUNNE. Facultad de Humanidades. UNNE. Año 15. № 30. Julio - Diciembre 2018. ISSN 1668-5180 Resistencia, Chaco.

En: http://hum.unne.edu.ar/revistas/geoweb/default.htm 
Revista Geográfica Digital. IGUNNE. Facultad de Humanidades. UNNE. Año 15. № 30.

Julio - Diciembre 2018. ISSN 1668-5180 Resistencia, Chaco

Tabla 2. Comparación de los eventos pluviométricos ocurridos en 2012 y 2017.

\begin{tabular}{|l|l|l|}
\hline Caracterización Pluviométrica & $\mathbf{2 0 1 2}$ & $\mathbf{2 0 1 7}$ \\
\hline Intensidad (IEP) & $-1,4$ & 0,5 (Verano 1,5) \\
\hline Tipo de evento & Seco & Normal - Verano muy húmedo \\
\hline Duración & 13 meses & 16 meses \\
\hline Precipitación anual & $490,6 \mathrm{~mm}$ & $671,2 \mathrm{~mm}$ - Verano $>100 \mathrm{~mm}$ \\
\hline Tormentas torrenciales & 3 & 12 \\
\hline Días secos consecutivos & 46 & 24 \\
\hline
\end{tabular}

\section{Comparación de los resultados}

Los resultados de las reuniones y entrevistas se compararon con la variabilidad pluviométrica analizada para el período 1960-2017. Los AS percibieron cambios en el régimen de precipitaciones a escala regional que incluyeron un aumento de los procesos de erosión, mayor exposición a los anegamientos, mayor generación de cárcavas y cambios en las especies de peces (Tabla 3).

El período más seco fue entre 2008 y 2009 , pero 2008 fue el año más seco de los últimos cincuenta años. Por esta razón, los AS tuvieron una percepción de un período seco muy extenso. Debido a esto, las propuestas de adaptación englobaron medidas paliativas contra las sequías en lugar de contemplar posibles periodos húmedos con posibilidades de inundación (Tabla 3).

La variabilidad de las precipitaciones generó impactos socio-económicos. Por ejemplo, los pescadores tuvieron que navegar largas distancias mar adentro debido a la migración de especies y la disminución del recurso, lo que representa un aumento de riesgos y de costos. El aumento de temperatura junto con la disminución de las precipitaciones percibidas por los AS generó serios problemas para los bomberos debido a las dificultades para apagar los incendios y la exposición de las ciudades a un mayor riesgo de incendio. En invierno, en las ciudades costeras (Pehuen Co y Monte Hermoso), la actividad comercial depende de los ingresos de residentes permanentes de cada comunidad. Por ejemplo, un comerciante indicó: "Si un pescador no pesca, no comprará y mis ventas caerán". Si el clima mejora en el período de verano, las ventas de las tiendas locales disminuyen porque las personas pasan más tiempo en la playa y suspenden otras actividades alternativas. En este sentido, algunos comerciantes percibieron una extensión del "día de playa" debido a las temperaturas más altas como una situación negativa.

Al analizar las problemáticas según la división de las ciudades que se realizó para su estudio, se pudieron resumir en la tabla 4 las principales problemáticas que se presentan en las localidades del estuario y de la playa. De esta forma se observaron cambios considerables entre ambos eventos.

Los AS y TM que habitan en el estuario percibieron, durante el período seco, que se generó una reducción en el crecimiento de los peces, perjudicando las capturas y un aumento de las sequías con una intensificación extraordinaria de las tormentas de arena. Por otro lado, durante el evento lluvioso, los habitantes registraron un mayor número de tormentas eléctricas en el verano, lluvias torrenciales que generaron el anegamiento de algunos barrios y la reaparición de peces fuera de su época habitual.

Por otro lado, la población notó en 2012 un mayor número de incendios y un aumento de los canales y barras en la playa que causaron un mayor riesgo para los bañistas. Para el evento húmedo (2017) identificaron una disminución del flujo de visitantes como consecuencia del aumento de las tormentas

Publicado en formato digital: Dra. M. Luján Bustos y Dr. Federico Ferrelli. PERCEPCIÓN DE LA VARIABILIDAD PLUVIOMÉTRICA EN LAS POBLACIONES COSTERAS DEL SUR DE LA PROVINCIA DE BUENOS AIRES (ARGENTINA): Una Propuesta Para el Diseño de Estrategias de Adaptación. Revista Geográfica Digital. IGUNNE. Facultad de Humanidades. UNNE. Año 15. № 30. Julio - Diciembre 2018. ISSN 1668-5180 Resistencia, Chaco.

En: http://hum.unne.edu.ar/revistas/geoweb/default.htm 
que causaron daños sobre los médanos y las playas. El mayor número de tormentas estuvo relacionado, según los AS, con una mayor cantidad de Sudestadas (Tabla 3).

Tabla 3. Principales problemáticas ambientales identificadas por los AS en cada una de las localidades en estudio.

\begin{tabular}{|c|c|c|}
\hline $\begin{array}{l}\text { Percepción de } \\
\text { los AS }\end{array}$ & 2012 & 2017 \\
\hline $\begin{array}{l}\text { General Daniel } \\
\text { Cerri, Ingeniero } \\
\text { White, Punta } \\
\text { Alta y Bahía } \\
\text { Blanca }\end{array}$ & $\begin{array}{l}\text { - Reducción en el crecimiento de los } \\
\text { peces perjudicando las capturas } \\
\text { - Aumento en la frecuencia e } \\
\text { intensidad de los períodos de } \\
\text { sequía } \\
\text { - Tormentas de arena }\end{array}$ & $\begin{array}{l}\text { - Aumento de tormentas } \\
\text { eléctricas } \\
\text { - Anegamientos de calles } \\
\text { - Aparición de peces fuera de } \\
\text { su época habitual }\end{array}$ \\
\hline $\begin{array}{c}\text { Monte } \\
\text { Hermoso y } \\
\text { Pehuen Co }\end{array}$ & $\begin{array}{l}\text { - Aumento de incendios } \\
\text { - Presencia de canales y barras en la } \\
\text { costa que generan peligros para } \\
\text { los bañistas (indica mayor cantidad } \\
\text { de sedimento) }\end{array}$ & $\begin{array}{l}\text { - Anegamientos de calles } \\
\text { - Disminución del flujo } \\
\text { turístico } \\
\text { - Daños en los médanos y } \\
\text { playas } \\
\text { - Aumento de tormentas } \\
\text { eléctricas (verano) } \\
\text { - Aumento de sudestadas } \\
\text { (erosión fuerte) }\end{array}$ \\
\hline
\end{tabular}

\section{Propuestas de adaptación}

A partir del conocimiento adquirido por sus vivencias, los AS delimitaron estrategias de adaptación. En los últimos 50 años, el Polo Industrial y el Puerto ubicados en el estuario de Bahía Blanca generaron importantes actividades comerciales, pesqueras e industriales. Sin embargo, para los AS esas acciones son las principales fuentes de problemas ambientales del área de estudio. Luego, la mayoría de los AS mencionó que el gobierno local debería encargarse de estos "asuntos" ambientales. Esta idea surgió porque la descarga de aguas residuales sin tratamiento en el estuario podría ser la principal fuente de contaminación (Zilio et al., 2013). Sin embargo, en la última década, las industrias redujeron fuertemente la contaminación y, cada año, las compañías pagan un impuesto al Comité Técnico Ejecutivo (ETC). El ETC tiene la responsabilidad de monitorear y controlar las condiciones ambientales del estuario de Bahía Blanca. Otra propuesta de los AS fue aumentar la coordinación entre el Organismo Provincial para el Desarrollo Sostenible (OPDS) y la Oficina Provincial de Pesca para mejorar los controles sobre los efluentes.

En Ingeniero White (Fig. 1), uno de los principales problemas percibidos está relacionado con la disminución de las pesquerías, por lo tanto, algunos proponen un control más profundo de las actividades pesqueras. Algunos pescadores mencionaron que "la Guardia Costera podría hacer este control, especialmente sobre los barcos de arrastre. El mismo control también podría hacerse por radar cuando grandes barcos pescan cerca de la costa". Sin embargo, el área de pesca está fuera del alcance del sistema de radar del puerto, lo que significa que la Guardia Costera debería tener más barcos patrullando el área o invertir en un nuevo sistema de radar.

Monte Hermoso (Fig. 1) fue la ciudad con más soluciones potenciales para los problemas identificados. Para abordar la reducción y la variabilidad de los recursos pesqueros, los pescadores estaban monitoreando la evolución de la población de mejillón y formularon un proyecto de ley para el cierre de la temporada de pesca en la zona durante tres años. Además, tenían un proyecto sobre certificación de la pesca artesanal (este proyecto incluía a los pescadores de Pehuen Co) para aumentar el valor agregado de su producción. También se encuentra en actividad reciente una planta

Publicado en formato digital: Dra. M. Luján Bustos y Dr. Federico Ferrelli. PERCEPCIÓN DE LA VARIABILIDAD PLUVIOMÉTRICA EN LAS POBLACIONES COSTERAS DEL SUR DE LA PROVINCIA DE BUENOS AIRES (ARGENTINA): Una Propuesta Para el Diseño de Estrategias de Adaptación. Revista Geográfica Digital. IGUNNE. Facultad de Humanidades. UNNE. Año 15. № 30. Julio - Diciembre 2018. ISSN 1668-5180 Resistencia, Chaco.

En: http://hum.unne.edu.ar/revistas/geoweb/default.htm 
de procesamiento de pesca artesanal que incluye un biodigestor para los desechos de procesamiento y la generación de energía. Del mismo modo crearon un proyecto educativo que se integra con las escuelas con el propósito de enseñar la separación de desechos.

La erosión de la playa es el problema más importante en Pehuen Co (Fig. 1). Por lo tanto, los AS propusieron obstruir los efectos de las olas a través de rompeolas, rocas, murallones de piedras y estructuras blandas sobre el frente costero, la mayoría de ellos expreso: "No importa que se haga pero es necesario hacer algo ya".

\section{DISCUSIÓN Y CONCLUSIONES}

La variabilidad de la precipitación podría producir eventos climáticos extremos. Existe evidencia de que estos ocurren a escala mundial y en las últimas décadas han sido más intensos, frecuentes y duraderos (Parry et al., 2009, IPCC 2012). Se reconoce que el conocimiento científico y el aporte de los AS y TM desempeñan un papel indispensable para diseñar estrategias de adaptación a los efectos negativos de la alternancia de eventos secos y húmedos (Berkes 2009, Nilsson y Gerger Swartling 2009). Los métodos basados en entrevistas y la investigación de acción participativa, como los aplicados en este estudio, se han empleado para comprender el estado de los ecosistemas en el pasado y las diferentes percepciones de sus habitantes (Sáenz-Arroyo et al., 2005a; 2005b; Bunce et al. al. 2008; Lozano-Montes et al., 2008; Moerlein y Carothers 2012).

Por otro lado, los eventos de erosión, de sedimentación, el aumento del nivel del mar están relacionados con la expansión de la población humana (Defeo y McLachlan 2009, Revell et al., 2011). Esto podría afectar la calidad y disponibilidad de los hábitats de las especies y el acceso a las poblaciones de peces (Moerlein y Carothers 2012; Defeo et al., 2013). Este último punto fue identificado por los pescadores de Ingeniero White, Pehuen Co y Monte Hermoso. También es interesante que en cuatro ciudades (Pehuen Co, Ingeniero White, General D. Cerri y Villa del Mar) la población consideraba la variabilidad pluviométrica un problema grave, pero seguía siendo una prioridad baja en relación con otros problemas ambientales como la contaminación. Esto también encontró Leiserowitz (2006) cuando observo que la variabilidad climática actualmente carece de sentido de urgencia para los AS y TM.

Los resultados obtenidos en este estudio son consistentes con las investigaciones que consideraron el vínculo entre la percepción social y la variabilidad pluviométrica. En algunos de ellos, la intervención de los AS generó cuatro temas relacionados con la variabilidad climática y las prácticas de subsistencia: 1) cambio ambiental físico, 2) cambios en la distribución y abundancia de peces, 3) efectos climáticos impredecibles en la pesca tradicional y 4) socioeconómicos y culturales cambio (Moerlein y Carothers, 2012). Cada uno de estos puntos se identificaron y trabajaron con los AS durante los talleres de este trabajo. Otros autores, también han realizado estudios sobre el análisis de percepciones en diferentes sitios, por ejemplo, Semenza et al. (2008) estudiaron la percepción en Houston y Portland sobre la conciencia, la preocupación y el cambio de comportamiento relacionado con el cambio climático.

Se considera importante que la mayoría de la población local, así como la población mundial, haya escuchado sobre el cambio climático global o el calentamiento global y la variabilidad climática. La mayoría de los AS reconoció la importancia de la variabilidad pluviométrica sobre el desarrollo de sus principales actividades económicas. Cabe destacar que su percepción impacta sobre sus actividades económicas y políticas de conservación ambiental. Este aspecto coincidió con la información que se obtuvo de los talleres relacionados con las diferentes expresiones de comerciantes, guardavidas y pescadores y cómo tratan de proteger sus recursos (por ejemplo, especies para la pesca, médanos, entre otros). Finalmente, se tuvo en cuenta que los efectos de la variabilidad pluviométrica podría disminuir con los cambios de comportamiento y las medidas de política activa, tal como expresaron Evans y Stevens (2007) y Semenza et al. (2008). En este sentido, los principales problemas detectados en el área de estudio son las distancias entre cada ciudad, la falta de comunicación en algunos de ellos, los diferentes partidos administrativos (con sus propias políticas) y un contexto de historia cultural que afecta el consenso entre ellos. Estos aspectos se refieren a la generación y aplicación de políticas ambientales, sociales y económicas para cada ciudad y región. Además, se ha

Publicado en formato digital: Dra. M. Luján Bustos y Dr. Federico Ferrelli. PERCEPCIÓN DE LA VARIABILIDAD PLUVIOMÉTRICA EN LAS POBLACIONES COSTERAS DEL SUR DE LA PROVINCIA DE BUENOS AIRES (ARGENTINA): Una Propuesta Para el Diseño de Estrategias de Adaptación. Revista Geográfica Digital. IGUNNE. Facultad de Humanidades. UNNE. Año 15. № 30. Julio - Diciembre 2018. ISSN 1668-5180 Resistencia, Chaco.

En: http://hum.unne.edu.ar/revistas/geoweb/default.htm 
demostrado que cuando los AS aumentaban su participación en la gestión de sus recursos, la aplicación de políticas ambientales tiene más éxito (Johnson et al., 2002).

Particularmente, para el área de estudio, no hubo anteriormente vínculo para con los AS y TM para que expresen sus opiniones sobre los efectos de la variabilidad pluviométrica y sus propuestas de adaptación a sus efectos. En ese sentido, los talleres realizados durante este estudio promovieron la creación de un marco en el que tuvieron lugar las interrelaciones entre AS, TM y la comunidad científica, en el camino de diálogos largos y constructivos. Los primeros (AS) son los que se ocupan de las cuestiones ambientales día a día. Los segundos (TM) son aquellos que proponen soluciones que no siempre se ajustan a las necesidades de la sociedad o los problemas ambientales reales. Mientras que los últimos son la clave para identificar problemas ambientales y proporcionar soluciones viables a estos, sobre la base de datos y experiencias previas de los otros dos actores.

Del estudio realizado, resulta sugerente que cualquier política o política pública que se quiera implementar dentro del área de estudio, con el fin de promover la adaptación a la variabilidad pluviométrica, considere la percepción de la población para asegurar su éxito. Esto se debe al hecho de que las percepciones de los AS son, en su gran mayoría, confirmadas por los datos medidos. Por lo tanto, es necesario tomar conciencia de que los TM deben adoptar políticas tendientes a adaptarse a posibles sequias e inundaciones.

En resumen, se aboga por la necesidad de centrarse en casos particulares planteados localmente por los AS y las posibles soluciones que podrían construirse a su alrededor. Por supuesto los AS no siempre presentan la solución más adecuada o viable para los problemas ambientales, por este motivo es necesario trabajar con ellos en forma conjunta de forma interdisciplinaria para lograr la inclusión de políticas de mitigación o adaptación.

\section{AGRADECIMIENTOS}

Queremos agradecer en primer lugar la buena predisposición de los habitantes del área de estudio para brindarnos información. Además, agradecemos el financiamiento otorgado por el proyecto COMET-LA (Community-based Management of Environmental Challenges in Latin America), la Universidad Nacional del Sur (UNS) y el Consejo Nacional de Investigaciones Científicas y Técnicas (CONICET). Finalmente, queremos destacar al Servicio Meteorológico Nacional (SMN) por facilitarnos la información meteorológica analizada en este estudio.

Publicado en formato digital: Dra. M. Luján Bustos y Dr. Federico Ferrelli. PERCEPCIÓN DE LA VARIABILIDAD PLUVIOMÉTRICA EN LAS POBLACIONES COSTERAS DEL SUR DE LA PROVINCIA DE BUENOS AIRES (ARGENTINA): Una Propuesta Para el Diseño de Estrategias de Adaptación. Revista Geográfica Digital. IGUNNE. Facultad de Humanidades. UNNE. Año 15. № 30. Julio - Diciembre 2018. ISSN 1668-5180 Resistencia, Chaco.

En: http://hum.unne.edu.ar/revistas/geoweb/default.htm 


\section{REFERENCIAS}

Aliaga, V.S., Ferrelli, F. y Piccolo, M.C. 2017. Regionalization of climate over the Argentine Pampas, International Journal of Climatology, 37 (S1): 1237-1247.

Barton, E.D., Lavín, M.F. y Trasviña, A. 2009. Coastal circulation and hydrography in the Gulf of Tehuantepec, Mexico, during winter, Continental Shelf Research, 29: 485-500.

Berkes, F. 2009. Indigenous ways of knowing and the study of environmental change, Journal of the Royal Society of New Zealand, 39(4):151-156.

Bolognesi, E., 1971. Análisis de criterios para clasificar totales mensuales de precipitación aplicados a series de República Argentina. Meteorología. Centro Argentino de Meteorólogos. Vol II $N^{0} 1,2$ y 3 . Buenos Aires.

Bohn, V.Y., Delgado, A.L., Piccolo, M.C. y Perillo, G.M.E. 2016. Assessment of climate variability and land use effect on shallow lakes in temperate plains of Argentina. Environmental Earth Science, 75: 1-15.

Bunce, M., Rodwell, L.D., Gibb, R., y Mee, L. 2008. Shifting baselines in fishers' perceptions of island reef fishery degradation. Ocean \& Coastal Management, 51:285-302.

Bustos, M.L., Piccolo, M.C. y Perillo, G.M.E. 2009. Cambios en la geomorfología de la playa de Pehuen Co debido a la actividad de las olas el 26 de julio de 2007. En: El territorio, las actividades económicas y la problemática ambiental en el Sudoeste Bonaerense: Universidad Nacional del Sur. Ediuns, Bahía Blanca, Argentina.

Bustos, M. L., Perillo, G.M.E., Piccolo, M.C. 2017. Dinámica de perfiles de playa en zonas con médanos frontales modificados en Pehuen Co (Argentina). Latin American Journal of Sedimentology and Basin Analysis, 23(2): 133-149.

Bustos, M.L., Ferrelli, F. y Piccolo, M.C. 2016. Diversificación de la actividad turística de balnearios: el caso de Pehuen Co, Argentina. Cuadernos de Turismo, 38 (2): 245-261.

Defeo, O. y McLachlan, A. 2009. Coupling between macrofauna community structure and beach type: a desconstructive meta-analysis. Marine Ecology Pregress Series 433: 29-41.

Defeo, O., Castrejón, M. Ortega, L. Kuhn, A.M. Gutiérrez, N. y Castilla, J.C. 2013. Impacts of climate variability on Latin American Small-scale fisheries. Ecology and Society, 18 (4): 30.

Edwards, C.D.C., McKee, T.B., Doesken, N.J. y Kleist, J. 1997. Historical analysis of drought in the United States. 7th Conference on Climate Variations, 77th AMS Annual Meeting, 27.

Evans, A., y Stevens, D. 2007. Climate change: the state of the debate. The London Accord. Available at http://www.cic.nyu.edu/internationalsecurity/docs/LondonAccordclimatepaper.pdf.

Ferrelli, F., Bohn, V. y Piccolo, M.C. 2012. Variabilidad de la precipitación y ocurrencia de eventos secos en el sudoeste de la provincia de Buenos Aires (Argentina). IX Jornadas Nacionales de Geografía Física, 15- 28.

Ferrelli, F., Bustos, M.L. y Piccolo, M.C. 2017. Variabilidad climática y sus efectos: aportes para el ordenamiento territorial de la costa norte del estuario de Bahía Blanca (Argentina). Revista Universitaria de Geografía, 26 (1): 79-96.

Geilfus, F. 2002. 80 Herramientas para el desarrollo participativo: diagnóstico, planificación, monitoreo, evaluación / FransGeifus IICA, IPCC, 2007. - San José, C.R

Hayes, M., Svoboda, M., Wall, N. y Widhalm, M. 2011. The Lincoln declaration on drought indices: universal meteorological drought index recommended. Bull. Am. Meteorol. Soc., 92(4): 485.

Huamantinco Cisneros, M.A.; Fornerón, C.F. Piccolo, M. C. y Perillo, G.M.E. 2011. Análisis de tormentas en dos ambientes acuáticos durante el invierno de 2009 (Partido de Monte Hermoso, Argentina). Revista Geográfica del Instituto Panamericano de Geografía e Historia (IPGH): 9-21.

Huamantinco Cisneros, M.A. 2012. Efecto de la variabilidad climática del balneario Monte Hermoso sobre su geomorfología costera y el confort climático. Departamento de Geografía y Turismo. Universidad Nacional del Sur, Bahía Blanca, Argentina. Tesis doctoral, 262 p. Inédito.

IPCC. 2012. Summary for policymakers. Pages 3-21. En: C. B. Field, V. Barros, T. F. Stocker, D. Qin, D. J. Dokken, K. L. Ebi, M. D. Mastrandrea, K. J. Mach, G.-K. Plattner, S. K. Allen, M. Tignor, y P. M. Midgley (ed.), Managing the risks of extreme events and disasters to advance climate change

Publicado en formato digital: Dra. M. Luján Bustos y Dr. Federico Ferrelli. PERCEPCIÓN DE LA VARIABILIDAD PLUVIOMÉTRICA EN LAS POBLACIONES COSTERAS DEL SUR DE LA PROVINCIA DE BUENOS AIRES (ARGENTINA): Una Propuesta Para el Diseño de Estrategias de Adaptación. Revista Geográfica Digital. IGUNNE. Facultad de Humanidades. UNNE. Año 15. № 30. Julio - Diciembre 2018. ISSN 1668-5180 Resistencia, Chaco.

En: http://hum.unne.edu.ar/revistas/geoweb/default.htm 
adaptation. A Special Report of Working Groups I and II of the Intergovernmental Panel on Climate Change. Cambridge University Press, Cambridge, UK.

IPCC. 2013. Cambio Climático 2013: Bases físicas, Contribución del Grupo de Trabajo I al Quinto Informe de Evaluación del IPCC, Glosario.

Johnson, N., Ravnborg, H. M., Westermann, O. y Probst, K. 2002. User participation in watershed management and research. Water Policy 3(6): 507-520.

London, S., Recalde, M. Rojas, M. Zilio, M. Perillo, G. M.E. Bustos, M. L. Piccolo, M. C. Rodriguez, C. Fidalgo, G. Pascale, J. C. Berninsone, L. Huamantinco Cisneros, M. A. Vaquero, M. C. y Bordino, P. 2012. Stakeholders vision on social-ecological system situation in Argentina case study. Comet-LA Project, Seventh Framework Programme, Disponible en: http://www.cometla.net/sites/default/files/DELIVERABLE\%20ARGENTINA\%20FINAL.pdf

Lozano-Montes, H.M., Pitcher, T.J. y Haggan, N. 2008. Shifting environmental and cognitive baselines in the upper Gulf of California. Frontiers in Ecology and the Environment, 6:75-80.

Magrin et al, 2007

Merlotto, A. y Bértola, G.R. 2006. Evolución urbana y su influencia en la erosión costera en el balneario parque Mar Chiquita, Argentina. Papeles de Geografía, 47-48: 143-158.

Moerlein, K. J., y Carothers, C. 2012. Total environment of change: Impacts of climate change and social transitions on subsistence fisheries in Northwest Alaska. Ecology and Society 17 (1): 10.

Nilsson, A.E. y Gerger Swartling, A. 2009. Social learning about climate adaptation: global and local perspectives. Stockholm Environment Institute (SEI) Working Paper. SEI, Stockholm, Sweden. Disponible http://www.seinternational.org/mediamanager/documents/Publications/Policyinstitutions/social learnin g wp 091112

Orlowsky, B, Seneviratne, S.I. 2013. Elusive drought: uncertainty in observed trends and shortand long-term CMIP5 projections. Hydrol. Earth Syst. Sci., 17(5): 1765-1781.

Parry, M., Arnell, N. Berry, P. Dodman, D. Fankhauser, S. Hope, C. Kovats, S. Nicholls, R. Satterthwaite, D. Tiffin, R. y Wheller, T. 2009. Assessing the costs of adaptation to climate change: a review of the UNFCCC and other recent estimates. International Institute for Environment and Development and Grantham Institute for Climate Change, London, UK.

Penalba, O.C., Rivera, J.A. 2016. Regional aspects of future precipitation and meteorological drought characteristics over Southern South America projected by a CMIP5 multi-model ensemble. Int. J. Climatol. 36(2): 974-986.

Perillo, G.M.E. 1997. Evaluación de la vulnerabilidad de la costa argentina al ascenso del nivel del mar. PNUD/SECYT ARG/95/G/31, 62 p.

Peterson, T., Folland, C., Gruza, G., Hogg, W., Mokssit, A. y Plummer, N. 2001. Report on the activities of the working group on climate change detection and related rapporteurs. Geneva: World Meteorological Organization, $146 \mathrm{p}$.

Pratolongo, P., Salinero, G. y Perillo, G.M.E. 2006. Evolución de la línea de costa frente al balneario Pehuen Co, pcia. de Buenos Aires, entre los años 1969 y 1996 . VI Jornadas de Ciencias del Mar, Puerto Madryn (resumen)

Revell, D.L., Dugan, J.E. y Hubbard, D.M. 2011. Physical and ecological responses of sandy beaches to the 1997-98 El Niño. Journal of Coastal Research, 27: 718-730.

Rojas, M.L., Recalde, M.Y. London, S. Perillo, G.M.E. Zilio, M. I. y Piccolo, M.C. 2014. Behind the increasing erosion problem: The role of local institutions and social capital on coastal management in Argentina. Ocean \& Coastal Management, 93:76-87.

Sáenz-Arroyo, A., Roberts, C.M. Torre, J. y Cariño-Olvera, M. 2005a. Using fishers' anecdotes, naturalists' observations and grey literature to reassess marine species at risk: the case of the Gulf grouper in the Gulf of California, Mexico. Fish and Fisheries, 6:121-133.

Sáenz-Arroyo, A., Roberts, C. M. Torre, J. Cariño-Olvera, M. y Enríquez-Andrade, R. R. $2005 \mathrm{~b}$. Rapidly shifting environmental baselines among fishers of the Gulf of California. Proceedings of the Royal Society B: Biological Sciences, 272:1957-1962.

Publicado en formato digital: Dra. M. Luján Bustos y Dr. Federico Ferrelli. PERCEPCIÓN DE LA VARIABILIDAD PLUVIOMÉTRICA EN LAS POBLACIONES COSTERAS DEL SUR DE LA PROVINCIA DE BUENOS AIRES (ARGENTINA): Una Propuesta Para el Diseño de Estrategias de Adaptación. Revista Geográfica Digital. IGUNNE. Facultad de Humanidades. UNNE. Año 15. № 30. Julio - Diciembre 2018. ISSN 1668-5180 Resistencia, Chaco.

En: http://hum.unne.edu.ar/revistas/geoweb/default.htm 
Semenza, J.C., Hall, D. E. Wilson, D. J. Bontempo, B. D. Sailor, D. J. y George, L. A. 2008. Public perception of climate change. American Journal of Preventive Medicine, 35:479-487.

UNISDR (United Nations International Strategy for Disaster Reduction Secretariat), 2011. Global Assestement Report on Disaster Risk Reduction 2011: Revealing Risk, Redefining Development. Oxford.

Wang, X. L., Chen, H., Wu, Y., Feng, Y. y Pu, Q. 2010. New techniques for the detection and adjustment of shifts in daily precipitation data series. Journal of Applied Meteorology and Climatology, 49 (12), 2416-2436.

Zhang, X., Alexander, L., Hegerl, G.C., Jones, P., Tank, A.K., Peterson, T.C., Trewin, B. y Zwiers, F.W. 2011. Indices for monitoring changes in extremes based on daily temperature and precipitation data. Wiley Interdisciplinary Reviews: Climate Change 2 (6), 851-870.

Zhang, X.,Yang, F. 2013 RClimDex (1.1) user manual. Available at http: // cccma. Seos. Uvic. Ca /ETCCDI/software.shtml. (last access:14/04/2018).

Zilio, M.I., London, S. Perillo, G.M.E. y Piccolo, M.C. 2013. The social cost of dredging: The Bahia Blanca Estuary case. Ocean \& Coastal Management, 71:195-202.

Publicado en formato digital: Dra. M. Luján Bustos y Dr. Federico Ferrelli. PERCEPCIÓN DE LA VARIABILIDAD PLUVIOMÉTRICA EN LAS POBLACIONES COSTERAS DEL SUR DE LA PROVINCIA DE BUENOS AIRES (ARGENTINA): Una Propuesta Para el Diseño de Estrategias de Adaptación. Revista Geográfica Digital. IGUNNE. Facultad de Humanidades. UNNE. Año 15. № 30. Julio - Diciembre 2018. ISSN 1668-5180 Resistencia, Chaco.

En: http://hum.unne.edu.ar/revistas/geoweb/default.htm 